\title{
Structure of hydrogen-dosed graphene deduced from low electron energy loss characteristics and density functional calculations
}

\author{
U. Bangert, ${ }^{1, a)}$ C. T. Pan, ${ }^{2}$ R. R. Nair, ${ }^{2}$ and M. H. Gass ${ }^{3}$ \\ ${ }^{1}$ School of Materials, The University of Manchester, Manchester M1 7HS, United Kingdom \\ ${ }^{2}$ School of Physics and Astronomy, The University of Manchester, Manchester M1 7HS, United Kingdom \\ ${ }^{3}$ Laboratories, CCLRC Daresbury Laboratory, Warrington WA4 4AD, United Kingdom
}

(Received 19 October 2010; accepted 20 November 2010; published online 23 December 2010)

\begin{abstract}
We employed nanometer-scale spatially resolved electron energy loss spectroscopy to monitor the degree and localization of electronic passivation of graphene surfaces by using the $\pi$-plasmon as an indicator of $\mathrm{sp}^{2}$-bonding. Upon hydrogenation, the $\pi$-plasmon does not vanish, and a new feature at $7 \mathrm{eV}$ occurs in the spectra. This behavior conforms to the theoretical electron energy loss spectra derived from density functional calculations for partial hydrogenation, with benzene rings assuming a corrugated structure. The spatial distribution of the spectral features and atomic resolution Z-contrast images indicate absence of any long-range ordering in the hydrogenated areas. (C) 2010
\end{abstract} American Institute of Physics. [doi:10.1063/1.3526373]

Much effort is put into modifying and functionalizing graphene surfaces via doping and dosing. Hydrogenation is expected, and has been shown, to open a band gap in graphene. ${ }^{1}$ Many theoretical structural models have been put forward such as armchair geometries of the benzene rings under $\mathrm{H}$-attachment, introducing out-of-plane bond angles. Various bond angles have been suggested. ${ }^{2}$ A contraction of the projected interatomic distance would be and has consequently been observed in diffraction patterns. ${ }^{1}$ However, the absolute interatomic distance is expected to increase upon hydrogenation; furthermore, areas of increased projected bond length have also been observed in diffraction patterns. More sophisticated models have suggested twisted chair or boat configurations. ${ }^{2,3}$ The structure is far from being resolved and controversial experimental observations led to the dispute of whether hydrogenation occurs randomly in a disordered fashion, or whether it takes place in domains, and furthermore, how large such domains would be and to what degree ordering might take place.

The experiments reported here were conducted in a dedicated scanning transmission electron microscope (the $\mathrm{C}_{\mathrm{s}}$-corrected Daresbury SuperSTEM), employing probe sizes $\leq 2 \AA$, and equipped with an Enfina electron energy loss (EEL) spectrometer. Operating voltages of 80 and $100 \mathrm{keV}$ were used; the instrumental resolution for EEL spectroscopy is $0.28 \mathrm{eV}$. Highly spatially resolved EEL analysis is important in this study since uncontaminated graphene, obtained by all known methods, only constitutes patches of several tens of nanometers in diameter; these are surrounded by contaminated areas, mostly due to hydrocarbons and fragments of further graphene layers. Analyses employing larger electron beams monitor the superposition of signals from pristine and contaminated areas. Since contaminants significantly alter the plasmon characteristics, nanometer EEL resolution is required in order to ascertain native graphene features.

Graphene samples were obtained using micromechanical cleavage and identification of graphene was performed using an optical microscope, followed by manual selection and deposition of flakes on transmission electron microscopy

${ }^{\text {a)} E l e c t r o n i c ~ m a i l: ~ u r s e l . b a n g e r t @ m a n c h e s t e r . a c . u k . ~}$
(TEM) grids, and in a number of samples, by electron beam lithography and etching steps in order to create a userdefined sample support. The method has been extensively reported, e.g., in Ref. 4. Hydrogen dosing was achieved by exposure to a hydrogen plasma, as detailed in Ref. 1 .

The collective movement of the graphitic $\pi$-electrons is a sensitive indicator of $\mathrm{sp}^{2}$-bonding, and hence of the electronic structure in graphitic systems, and can therefore be used to monitor the degree and localization of electronic passivation of graphene surfaces, which would occur through hydrogen dosing. $\pi$-plasmon characteristics have been extensively studied in graphite. ${ }^{5}$ The observed low plasmon energy in a single sheet ${ }^{6}$ is an indication of a strong in-plane confinement; this has been discussed for low energy plasmons. $^{7,8}$ An EEL spectrum showing the $\pi$ - and $\sigma$-plasmon of graphene at 4.7 and $14.7 \mathrm{eV}$ is shown in the gray curve in Fig. 1(b).

Hydrogenation ought to passivate the $\pi$-bonds and cause formation of $\mathrm{sp}^{3}$ bonds instead, and hence dramatically diminish the occurrence of the $\pi$-plasmon in comparison to unhydrogenated graphene. However, the $\pi$-plasmon in graphene exposed to a hydrogen plasma [marked by the rectangle (yellow online) in the black curve, Fig. 1(b)] is nearly undiminished in areas, which, at the same time, show an $\mathrm{H}$ ground state excitation peak at $\sim 13.2 \mathrm{eV}$ [dashed oval; purple online in Fig. 1(b)]. The EEL spectrum of the hydrogen K-edge in hydrogenated graphene has been published elsewhere. ${ }^{9}$ Furthermore, Fig. 1(b) shows a new feature at $\sim 7 \mathrm{eV}$ (oval; turquoise online). This $7 \mathrm{eV}$ feature only occurs in hydrogenated graphene and is similar to a feature occurring on unpassivated diamond surfaces, ${ }^{10}$ which have undergone reconstruction of their dangling bonds into $\pi$-bonded chains.

It is reasonable to presume that coexistence of the $\pi$-plasmon and the $13.2 \mathrm{eV}$ signal means that hydrogenation is not complete, i.e., not every $\mathrm{C}$ atom is bonded to an $\mathrm{H}$ atom. Figures $1(\mathrm{~d})-1(\mathrm{~g})$ show a high angle annular dark field (HAADF) image, the spatial distribution of the $\mathrm{H}$ ground state signal, the $\pi$-plasmon at $4.7 \mathrm{eV}$, and the $7 \mathrm{eV}$ feature, respectively. Since all three signals have near-identical distributions, this would then indicate that nonhydrogenated and 


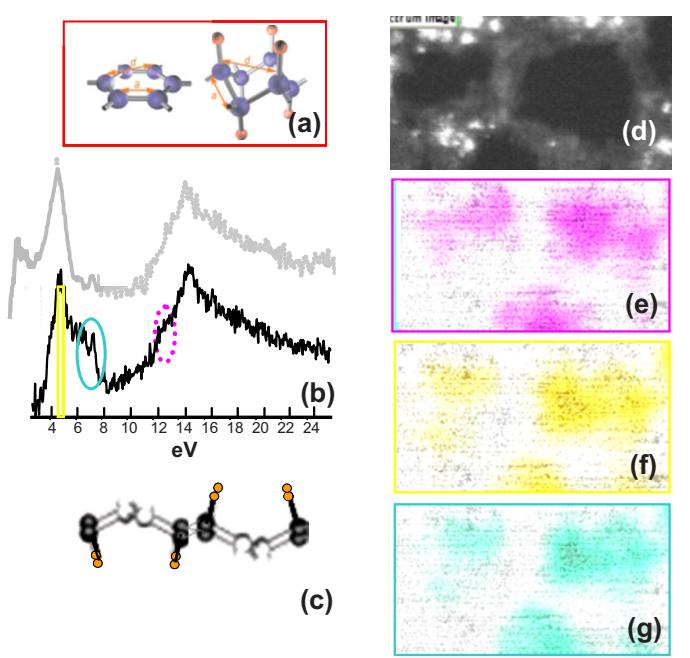

FIG. 1. (Color online) (a) Benzene ring in graphene (left) and armchair configuration assumed after hydrogenation $\mathrm{H}$ (small circles) bonded to $\mathrm{C}$ atoms (big black circles) and non-hydrogenated $\mathrm{C}$-atoms (big white circles) in right sketch. (b) The EEL spectra of hydrogenated graphene (black) showing the $\pi$-plasmon (marked by rectangle; yellow online), the $7 \mathrm{eV}$ feature (circled by solid line; turquoise online), the $\mathrm{H}$ ground state transition (circled by dashed line; purple online), and the graphene $\sigma$-plasmon (at $\sim 15 \mathrm{eV}$ ); the gray spectrum (vertically displaced) shows a low loss spectrum of pristine graphene for comparison. (c) Possible model for the arrangement of $\mathrm{H}-\mathrm{C}$ bonds in coexistence with $\pi$-bonded chains (white atoms). (d) HAADF STEM image of hydrogenated graphene; the dark areas are "windows" free from deposits, the gray/white features are hydrocarbon deposits. (e) Intensity distribution of the $\mathrm{H}$ ground state transition extracted from the EEL spectrum. (f) and (g) display similar intensity distributions as in (e) but for the $\pi$-plasmon and the $7 \mathrm{eV}$ transition. Note the similarity in the distributions in (e)-(g) and the absence of features in areas of hydrocarbon deposits. The frame width in (d)-(g) is $30 \mathrm{~nm}$.

hydrogenated bonds coexist in close proximity; hence, complete hydrogen coverage is not achieved. It should be noted that the brighter regions in the HAADF image [Fig. 1(d)] represent hydrocarbon contamination. The $\mathrm{H}$ ground state signal here is shifted to lower values, while the $\pi$-plasmon is shifted to higher energy values, and the $7 \mathrm{eV}$ feature is missing; hence, there is an absence of signal intensity in the areas in Figs. 1(e) $-1(\mathrm{~g})$.

In order to support the above interpretation of the EEL spectroscopy observations and to reproduce the EEL spectra theoretically, density functional theory (DFT) calculations using the WIEN2K code have been performed. WIEN2K is an $a b$ initio code with all-electron full potentials and uses the general gradient of Perdew-Burke-Enzerhof 96 with a mixed linearized augmented plane wave/augmented plane wave + local orbitals (mixed LAPW / APW + lo). ${ }^{11,12}$ Previous band structure calculations of fully hydrogenated graphene using CASTEP, another ab initio code with pseudopotentials, have shown that graphane is a wide band gap semiconductor with an $\sim 3.7 \mathrm{eV}$ band gap and that $\mathrm{C}$ atoms are displaced from planar geometries by $\mathrm{H}$ atoms. ${ }^{2}$

WIEN2K has not been previously applied to graphene calculations. Tests run on graphite and pure graphene show results identical to those from our previous $a b$ initio calculations using the AIMPRO code, ${ }^{6}$ where plasmons were found at $6-7 \mathrm{eV}(\pi$-plasmon $)$ and $\sim 26 \mathrm{eV}(\pi+\sigma$-plasmon $)$ in graphite and redshifted to 4.7 and $14.7 \mathrm{eV}$, respectively, in graphene. Freestanding graphene was simulated by separating the sheets beyond the range of interaction, i.e., by building supercells with up to $7.5 \mathrm{~nm}$ sheet separation. It was found that interaction ceased and calculations already con- verged sufficiently at separations of 3 and even $1 \mathrm{~nm}$. Simulations with a series of $\mathrm{K}_{\max }$ values and $\mathrm{K}$-point numbers were run to ascertain the convergence criteria. ${ }^{13-15}$ For graphene, the optimal parameters for our simulations were found as follows: $\mathrm{K}_{\max }=4$, muffin tin radius $\mathrm{R}_{\text {carbon }}=1.3426$, K-point number $=2000$, and layer spacing $\mathrm{C}=15 \AA$ (we kept the same K-point density for different layer spacings); for graphane, $\mathrm{K}_{\max }=4, \mathrm{R}_{\text {carbon }}=1.33, \mathrm{R}_{\text {hydrogen }}=0.72, \mathrm{~K}$-point number $=2000$, and layer spacing $\mathrm{C}=15 \AA \mathrm{A}$.

Figure 2(a) shows the calculated (black solid line) and experimental (dashed line; purple online) low loss spectra of graphene, demonstrating excellent mutual agreement. It should be noted that the $\pi$-plasmon intensity drops to zero or to background noise at $\sim 8 \mathrm{eV}$. There is also a prepeak at $\sim 2 \mathrm{eV}$ in the experimental data, which was retrieved after applying the Richardson-Lucy deconvolution algorithm; ${ }^{16,17}$ this prepeak is notably observed in the calculated spectra. The latter is obtained for sheets made up of flat benzene rings with $5.5 \mathrm{~nm}$ spacing. Interestingly, the curves are qualitatively very similar for separated sheets with benzene rings in corrugated (armchair) geometry (precise spectra not shown). Thus, it appears that the $\pi$-electron system also exists in single sheets with nonplanar geometries.

The following discussion will concentrate on the $\pi$-plasmon region, although calculations show spectra up to $35 \mathrm{eV}$. The $\pi+\sigma$-plasmon, even though differing in detail from calculation to calculation, occurs throughout at an energy of roughly $15 \mathrm{eV}$. As mentioned before, the experimental spectra of hydrogenated graphene exhibit a $\pi$-plasmon and an additional peak at $7 \mathrm{eV}$. The prepeak at $2 \mathrm{eV}$ has subsided. Figure 2(b) shows the evolution of the $\pi$-plasmon in fully hydrogenated graphene with increasing corrugation angle. Interestingly, flat graphane, similar to graphene, possesses a $\pi$-plasmon [Fig. 2(b), 90 , black solid curve]. However, its energy is slightly higher than experimentally observed. The $\pi$-plasmon has diminished for a corrugation angle of $99^{\circ}$ [Fig. 2(b), fine-dotted line; red online] and has nearly disappeared for corrugation angles larger than $107.8^{\circ}$ [Fig. 2(b)]. A feature at $\sim 6-7 \mathrm{eV}$ emerges at the rise of a (presumably) pure $\sigma$-plasmon, which sits at an energy slightly higher than the $\pi+\sigma$-plasmon in graphene. Hence, $\mathrm{sp}^{3}$ character does become established in corrugated graphene when all bonds are used. It should be noted that Figs. 2(a) and 2(b) show the in-plane plasmon component. Figures 2(c) and 2(d) show plasmon calculations of the inplane and out-of-plane plasmon components for different hydrogen coverages for a sheet corrugation angle of $114^{\circ}$ (as deduced from diffraction experiments ${ }^{1}$ ). The out-of-plane component does not have a $\pi$-plasmon for any fraction of hydrogen coverage [Fig. 2(d)]. Figure 2(e) shows the spectra resulting from summing the in-plane and out-of-plane components. This represents the experimental condition most accurately because the most likely electron scattering vector would produce a plasmon k-vector at $45^{\circ}$ to the graphene sheet, thus producing plasmons with approximately equal amounts of in-plane and out-of-plane components. The overlaid experimental spectrum fits the $33 \%$ hydrogenation case [Fig. 2(e), dotted green curve] rather well. (In contrast, the out-of-plane component does not add significantly to the shape of the $\pi$-plasmon in flat, pristine graphene. ${ }^{6}$ ) Models to describe possible structural scenarios are shown in the inset. It becomes clear that corrugation is essential to produce the $\sim 7 \mathrm{eV}$ feature, as is occurrence of $\mathrm{sp}^{3}$ character. In 
(a)
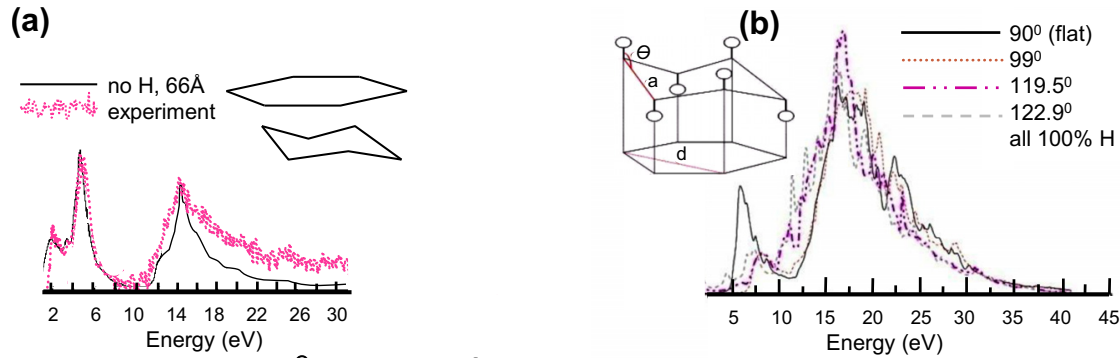

(c)
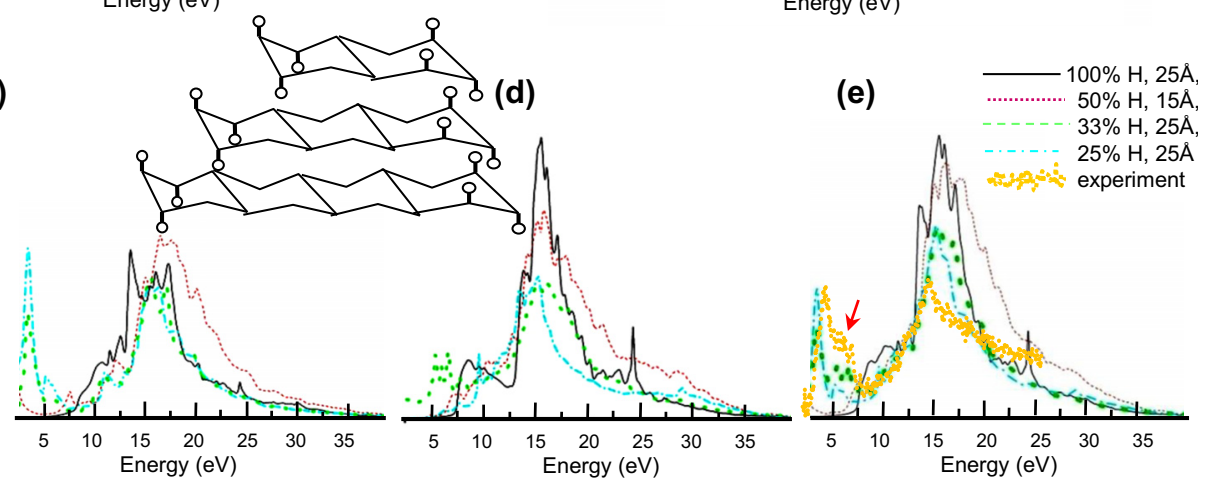

FIG. 2. (Color online) WIEN2K calculations of low loss EEL spectra for (a) nonhydrogenated graphene in flat and corrugated geometry for the in-plane plasmon component $(\boldsymbol{q} \| \boldsymbol{a})$ with overlaid experimental spectrum. Note the agreement in the preplasmon structure. (b) Fully hydrogenated graphene with varying corrugation angle $\theta$, again, for $(\boldsymbol{q} \| \boldsymbol{a})$. (c) and (d) show the calculated in-plane $(\boldsymbol{q} \| \boldsymbol{a})$ and out-of-plane $(\boldsymbol{q} \| \boldsymbol{c})$ plasmon components for corrugated graphene $\left(\theta=114^{\circ}\right)$ with different hydrogen contents (given in \% in the legend; model structures are shown in the sketch). (e) shows the sum of spectra from (c) and (d), representing the realistic situation with nearly equal contributions of both components. The dotted spectrum (orange online) overlaid in (e) is an experimental spectrum of a hydrocarbon-free region in $\mathrm{H}$-dosed graphene. Note the disappearance of the preplasmon feature and the occurrence of an extra peak at $\sim 7 \mathrm{eV}$. The distances given in $\AA$ in the legends are the separation distances of the graphene sheets or the supercell dimension along the c-axis.

order to preserve $\pi$-bonding, however, the corrugation angles have either to be small (as would be the case for strained graphene, if it is hydrogenated mainly on one surface $^{3}$ ) or unhydrogenated areas have to be present. Alternatively or simultaneously, hydrogenated patches could be interleaved with pure graphene areas.

HAADF images of pristine and hydrogenated graphene are shown in Figs. 3(a) and 3(b), respectively. We note that the benzene rings (bright hexagons) are clearly visible and undistorted throughout the image area in Fig. 3(a). Brighter patches in the image contrast arise from $\mathrm{C}$ adatoms. ${ }^{9}$ In the image of Fig. 3(b), benzene rings do not have the same regular appearance. The random variations in their contrast lead us to conclude that there is no long-range ordering of hydrogenated and unhydrogenated areas, and neither is there evidence of uniform corrugation. This conforms to the above conclusions of the coexistence of flat (unhydrogenated) benzene rings and benzene rings that have undergone out-ofplane distortions due to hydrogen bonded to the surface, a
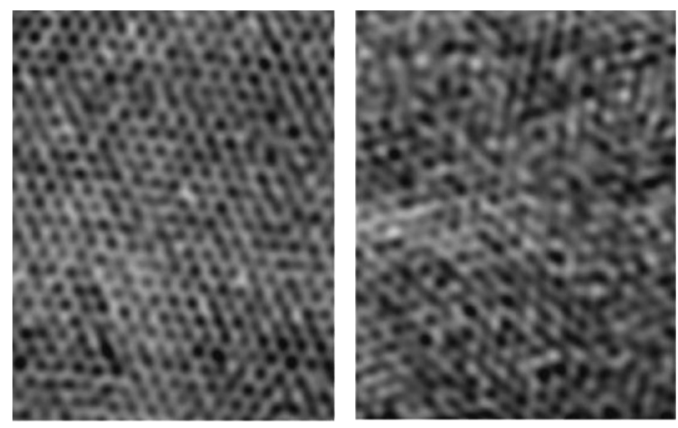

FIG. 3. Atomic resolution HAADF images of pristine (left) and hydrogenated (right) graphene. The frame width is $3 \mathrm{~nm}$. result derived from experimental and calculated EEL spectra showing a non-vanishing $\pi$-plasmon and occurence of a new feature at $7 \mathrm{eV}$.

${ }^{1}$ D. C. Elias, R. R. Nair, T. M. G. Mohiuddin, S. V. Morozov, P. Blake, M. P. Halsall, A. C. Ferrari, D. W. Boukhvalov, M. I. Katsnelson, A. K. Geim, and K. S. Novoselov, Science 323, 610 (2009).

${ }^{2}$ J. O. Sofo, A. S. Chaudhari, and G. D. Barber, Phys. Rev. B 75, 153401 (2007).

${ }^{3}$ K. Xue and Z.-P. Xu, Appl. Phys. Lett. 96, 063103 (2010).

${ }^{4}$ T. J. Booth, P. Blake, R. R. Nair, D. Jiang, E. W. Hill, U. Bangert, A. Bleloch, M. Gass, K. S. Novoselov, M. I. Katsnelson, and A. K. Geim, Nano Lett. 8, 2442 (2008).

${ }^{5}$ K. Zeppenfeld, Z. Phys. 243, 229 (1971).

${ }^{6}$ T. Eberlein, U. Bangert, R. R. Nair, R. Jones, M. H. Gass, A. L. Bleloch, K. S. Novoselov, A. K. Geim, and P. R. Briddon, Phys. Rev. B 77, 233406 (2008).

${ }^{7}$ M. Jablan, H. Buljan, and M. Soljačić, Phys. Rev. B 80, 245435 (2009).

${ }^{8}$ E. H. Hwang and S. Das Sarma, Phys. Rev. B 75, 205418 (2007).

${ }^{9}$ U. Bangert, M. H. Gass, A. L. Bleloch, R. R. Nair and J. L. Eccles, Phys. Status Solidi A 206, 2115 (2009).

${ }^{10}$ U. Bangert, R. Barnes, L. S. Hounsome, R. Jones, A. T. Blumenau, P. R. Briddon, M. J. Shaw, and S. Öberg, Philos. Mag. 86, 4757 (2006).

${ }^{11}$ M. Nelhiebel, P. H. Louf, P. Schattschneider, P. Blaha, K. Schwarz, and B. Jouffrey, Phys. Rev. B 59, 12807 (1999).

${ }^{12}$ P. Blaha, K. Schwarz, G. K. H. Madsen, D. Kvasnicka, and J. Luitz, WIEN2K: An augmented plane wave+local orbital program for calculating crystal properties, Karlheinz Schwarz, Techn, Universität Wien, Austria, 2001.

${ }^{13}$ R. J. Nicholls and A. J. Scott, J. Phys.: Conf. Ser. 126, 012038 (2008).

${ }^{14}$ C. R. Seabourne, A. J. Scott, R. Brydson, and R. J. Nicholls, Ultramicroscopy 109, 1374 (2009).

${ }^{15} \mathrm{~S}$. Cottenier, Density functional theory and the family of (L)APWmethods: A step-by-step introduction, Instituut voor Kern-en Stralingsfysica, K. U. Leuven, Belgium, 2002, http://www.wien2k.at/reg_user/ textbooks.

${ }^{16}$ W. H. Richardson, J. Opt. Soc. Am. 62, 55 (1972).

${ }^{17}$ L. B. Lucy, Astron. J. 79, 745 (1974). 